\title{
CÁNCER DE PIEL Y RADIACIÓN SOLAR: EXPERIENCIA PERUANA EN LA PREVENCIÓN Y DETECCIÓN TEMPRANA DEL CÁNCER DE PIEL Y MELANOMA
}

\author{
Carlos Sordo ${ }^{1,2, a}$, César Gutiérrez $3, b$
}

RESUMEN

\begin{abstract}
La excesiva exposición a la radiación solar, específicamente la ultravioleta (RUV), ha sido causa de diferentes enfermedades, en especial de cáncer de piel. En 1995 el Círculo Dermatológico del Perú realizó la primera "Campaña de Educación, Prevención y Detección Temprana de Cáncer de Piel y Melanoma" denominada el "Día del Lunar". Fue oficializada por el Ministerio de Salud, y cuenta con la participación del Seguro Social de Salud (EsSalud). Es una campaña gratuita que se realiza cada año a nivel nacional, en ella, desde 1995 al 2011 se atendieron a 118092 personas, en 76 sedes distribuidas en 18 ciudades de todo el país, en el 2,8\% de estas se pudo identificar alguna lesión cutánea sospechosa de malignidad, de las cuales el $64,9 \%$ correspondió a carcinoma basocelular, $26,7 \%$ a melanoma cutáneo y $8,4 \%$ a carcinoma espinocelular. Estas campañas destacan en importancia no solo por la práctica asistencial en ellas realizada, sino por las actividades educativas orientadas a fomentar una cultura de prevención en favor de las poblaciones más vulnerables. Finalmente, consideramos que es fundamental seguir educando a la población en la prevención del cáncer de piel, crear conciencia en las autoridades para que participen activamente en la realización de estas actividades, además de solicitar a todos los médicos que se sumen coordinadamente a este esfuerzo para seguir avanzando y mejorar lo logrado en beneficio de nuestro país.
\end{abstract}

Palabras clave: Neoplasias cutáneas; Melanoma; Prevención; Diagnóstico precoz (fuente: DeCS BIREME).

\section{SKIN CANCER AND SUN RADIATION: PERUVIAN EXPERIENCE IN THE PREVENTION AND EARLY DETECTION OF SKIN CANCER AND MELANOMA}

\begin{abstract}
The excessive exposure to sun radiation, especially to ultraviolet radiation (UV), has led to various diseases, in particular to skin cancer. In 1995, the Peruvian Dermatological Association conducted the first "Campaign for Education, Prevention and Early Detection of Skin Cancer and Melanoma" called "Mole's Day". The Ministry of Health has turned it into an official event, and the Health Social Security (EsSalud) also participates. This is a free campaign that takes place every year nationwide. 118,092 people attended from 1995 to 2011 in 76 sites distributed in 18 cities throughout the country. A cutaneous lesion were malignancy was suspected was identified in $2.8 \%$ of people attending, out of which $64.9 \%$ corresponded to basal cell carcinoma, $26.7 \%$ to cutaneous melanoma, and $8.4 \%$ to squamous cell carcinoma. These campaigns are highly important not only because of the assistance given, but also because of the educational activities aimed at promoting a prevention culture in favor of the most vulnerable populations. Finally, we believe it is important to continue educating the population on skin cancer prevention, to build awareness among the authorities so that they actively participate in the performance of these activities, and to ask all physicians to coordinately join this initiative, in order to continue growing, and to improve all that has been attained for the benefit of our country.
\end{abstract}

Key words: Skin neoplasms; Melanoma; Prevention; Early diagnosis (source: MeSH NLM).

\section{INTRODUCCIÓN}

Desde sus orígenes, el hombre ha identificado al sol como fuente de luz, calor y vida. En muchas de las culturas de la antigüedad se le asignó un rol divino asociado a la sanación ${ }^{(1)}$. Con el paso del tiempo, se comenzó a emplear los rayos solares en el tratamiento de diferentes enfermedades, entre ellas la artrosis, la tuberculosis, el raquitismo y la psoriasis ${ }^{(2)}$. La revolución industrial en Europa significó un periodo de gran

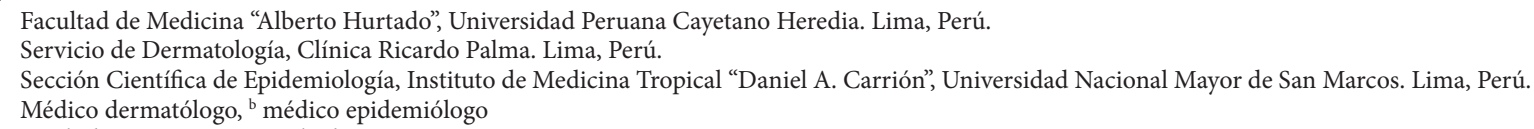

Citar como: Sordo C, Gutiérrez C. Cáncer de piel y radiación solar: experiencia peruana en la prevención y detección temprana del cáncer de piel y melanoma. Rev Peru Med Exp Salud Publica. 2013;30(1):113-7. 
desarrollo para la humanidad, e implicó un incremento en la contaminación ambiental en las ciudades, con lo cual aumentaron los casos de raquitismo. Conocedores de que la radiación ultravioleta (RUV) estimula la síntesis de vitamina $D$ y así se podía evitar la presentación de este mal, cada vez más gente buscaba exponerse al sol, buscando sus efectos beneficiosos. Estos hechos entre otros contribuyeron a difundir la idea que el bronceado era sinónimo de salud. Sin embargo, también se hizo evidente que la excesiva exposición a la radiación solar, específicamente a la RUV, estaba asociada con diferentes enfermedades, entre ellas el cáncer de piel, principalmente el melanoma maligno, el carcinoma de células escamosas y el carcinoma de células basales ${ }^{(3)}$.

Hoy conocemos, además, que la RUV se divide en radiación ultravioleta A (UVA), B (UVB) y C (UVC). La UVC es filtrada por la capa de ozono en la estratósfera y solo la $A$ y $B$ inciden en la superficie de la tierra y, por ende, en nuestra piel. La RUV constituye el $6 \%$ de la radiación solar recibida y, de esta, el 5-10\% corresponde a UVB y el $90-95 \%$ a UVA, igualmente se sabe que a mayor altitud se recibe mayor cantidad de radiación (4).

La exposición prolongada a la UVB es responsable del cáncer de piel dado que penetra superficialmente en la piel afectando la epidermis en donde daña directamente el ADN celular. Por su parte, la UVA penetra más profundamente, afectando la dermis, destruyendo las fibras elásticas y colágenas y condicionando envejecimiento, inmunosupresión, reacciones fotoalérgicas, reacciones fototóxicas debidas a medicamentos y generando radicales libres que dañan el ADN celular actuando de manera sinérgica con la UVB ${ }^{(5)}$. No se debe olvidar que la disminución de la capa de ozono, el efecto invernadero y el calentamiento global, aumentan la cantidad de RUV que se recibe todo el año, condicionando un aumento en la incidencia de cáncer de piel.

Puesto que el Perú no escapa a la situación mundial de cambios en las condiciones medio-ambientales que favorecen un mayor impacto negativo de la radiación solar, es importante aproximarnos al conocimiento del estado de las neoplasias cutáneas asociadas a la RUV. Así mismo, analizar algunos esfuerzos que se vienen realizando en la prevención del cáncer de piel, para de esta manera promover aún más los cuidados que deben tenerse frente a la RUV.

\section{EPIDEMIOLOGÍA DEL CÁNCER DE PIEL EN EL PERÚ}

En el Perú no se cuenta con datos sobre la incidencia de las neoplasias de piel, si bien el proyecto Globocan hace algunas estimaciones, estas se basan en modelos que toman en cuenta datos de países vecinos ${ }^{(6)}$. Sin embargo, la
Dirección General de Epidemiología $(D G E){ }^{(7)}$ ha realizado un análisis de la situación del cáncer a nivel nacional en base a la vigilancia epidemiológica de cáncer, encontrando que en el periodo comprendido entre los años 2006 y 2010 se registró un total de 5975 casos de cáncer de piel $(2744$ en varones y 3231 en mujeres) que representan el 6,6\% del total de cánceres registrados. Según este informe, el cáncer de piel ocupa el cuarto lugar de frecuencia a nivel nacional (superado por el cáncer de cérvix, estómago y mama). Las regiones donde la distribución proporcional del cáncer de piel respecto al total de neoplasias registradas es superior al promedio nacional $(6,6 \%)$ han sido La Libertad $(10,7 \%)$, Cajamarca $(9,5 \%)$, Madre de Dios $(9,2 \%)$, San Martín (8,0\%), Amazonas (7,9\%), Lima (7,9\%), Arequipa $(7,8 \%)$, Ayacucho $(7,3 \%)$ y Ucayali $(7,1 \%)$. Si bien estas proporciones no representan una medida de frecuencia de la enfermedad en la población, sí es importante resaltar la preponderancia del cáncer de piel en el sistema de vigilancia, y también que la mayoría de las regiones de la selva peruana presentan una distribución proporcional superior al promedio nacional. La DGE también señala que el año 2011 se registró un total de 1208 defunciones por neoplasias malignas de piel (725 en hombres y 483 en mujeres), lo que representó una tasa de mortalidad ajustada de 1,6 por 100000 (tasa de 2,1 por 100000 en hombres y 1,6 por 100000 en mujeres).

Otra información que también nos permite tener una aproximación al conocimiento de la situación de las neoplasias de piel en el país son las estadísticas registradas en el Departamento de Epidemiología y Estadística del Cáncer del Instituto Nacional de Enfermedades Neoplásicas (INEN), las cuales señalan que entre los años 2000 y 2011, se han atendido en el INEN 6048 casos de neoplasias de piel, que representan el 5,8\% del total de neoplasias atendidas. La evolución del número ha sido ascendente, pasando de

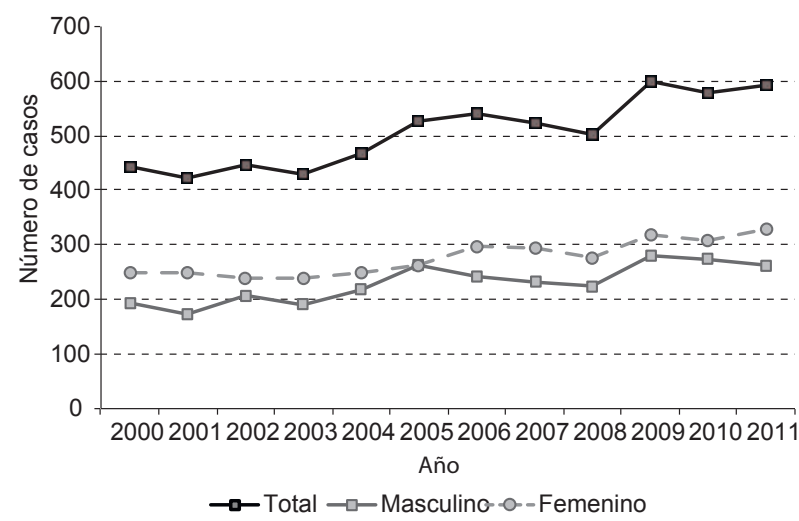

Figura 1. Casos de neoplasias cutáneas atendidas en el Instituto Nacional de Enfermedades Neoplásicas, 2000-2011.

Fuente de los datos: Departamento de Epidemiología y Estadística del Cáncer, Instituto Nacional de Enfermedades Neoplásicas (INEN). 
439 casos el año 2000 (191 hombres y 248 mujeres) a 592 casos el año 2011 (262 hombres y 330 mujeres) (Figura 1). Estos datos, si bien no corresponden a una incidencia, son un indicador de que el número de neoplasias cutáneas ha venido aumentado a nivel poblacional; puede haber algunas otras interpretaciones al mayor número de casos registrados, pero es razonable atribuir este incremento al aumento en la incidencia del melanoma ${ }^{(8)}$. Según grupos de edad, se ha observado que a mayor edad, mayor el número de casos atendidos; así, el 1,5\% de los casos de neoplasias cutáneas atendidos en el INEN entre 2000 y 2011 fueron pacientes menores de 20 años, el $7,7 \%$ tuvieron entre 20 y 39 años, el $26,8 \%$ entre 40 y 59 años y el $63,8 \%$ fueron personas de 60 años y más.

\section{EL DÎA DEL LUNAR: EXPERIENCIA PERUANA EN LA EDUCACIÓN PREVENCIÓN Y DETECCIÓN TEMPRANA DEL CÁNCER DE PIEL Y MELANOMA}

Los dermatólogos son los médicos especialistas que debido a su formación y entrenamiento están capacitados para identificar las lesiones cutáneas benignas, sospechosas de malignidad o malignas, durante el examen completo de la piel, para luego extirparlas o biopsiarlas, según convenga, a fin de remitirlas al patólogo para su estudio e identificación correspondiente y, si el caso lo requiere, derivar al paciente para su tratamiento oportuno.

Esta característica profesional fundamentalmente visual los llevó primero a conceptualizar una regla práctica a fin de identificar rápidamente lesiones sospechosas, acuñando la nemotecnia conocida como el ABCDE de los lunares; $A$ de asimetría, $B$ de bordes, $C$ de color, D de diámetro y $\mathrm{E}$ de elevación o evolución y, posteriormente, a difundir el concepto del autoexamen realizando campañas preventivas de despistaje de cáncer de piel. Las primeras campañas se realizaron en Estados Unidos de América y luego le siguieron las experiencias latinoamericanas de Chile, Argentina y Perú.

El Círculo Dermatológico del Perú (CIDERM) en el año 1995 realizó, por primera vez en nuestro país, la primera "Campaña de Educación, Prevención y Detección Temprana de Cáncer de Piel y Melanoma" denominada "Día del Lunar" siendo los objetivos, como su nombre lo indica, no solo detectar tempranamente las lesiones sospechosas de cáncer de piel sino educar a la población sobre los peligros que este conlleva, y los efectos negativos derivados de la sobreexposición al sol, creando conciencia y fomentando una cultura de prevención (9). Desde entonces el "Día del Lunar" se realiza a nivel nacional el primer domingo de febrero de cada año, de manera gratuita

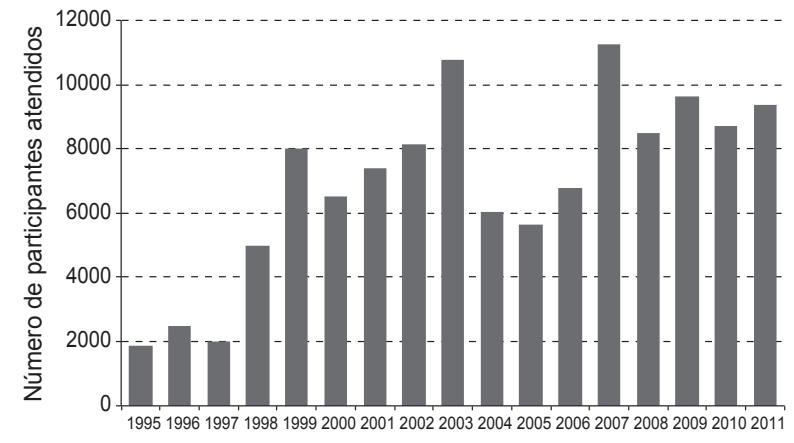

Figura 2. Atendidos en las campañas "El Día de Lunar", 19952011.

Fuente de los datos: Círculo Dermatológico del Perú (CIDERM).

gracias al trabajo voluntario de los dermatólogos peruanos y al apoyo de la industria farmacéutica; cuenta además con el auspicio del Ministerio de Salud y el Seguro Social de Salud (EsSALUD). La atención a los pacientes se realiza en hospitales y clínicas privadas en la fecha principal y a lo largo de la semana siguiente, y su difusión es a través de la prensa escrita, radial y televisiva. Debido al impacto que se produjo en los primeros cinco años y su importancia, el Ministerio de Salud oficializó el "Día del Lunar" con Resolución Ministerial 286-99 SA/DM, que fue publicada el sábado 19 de junio de 1999 en el Diario Oficial "El Peruano".

Según los registros del CIDERM, entre 1995 y 2011, se atendieron a 118092 personas, pasando de 1877 atendidos el año 1995 en doce sedes ubicadas solamente en Lima, a 9355 atendidos el año 2011 en 76 sedes distribuidas en 18 ciudades de todo el país (Figura 2). La mayor proporción de participantes han sido mujeres $(66,0 \%)$. El promedio de edad ha sido $41,4 \pm 20,4$ años, con un rango que va desde participantes menores de un año hasta personas mayores de 90. Los participantes varones han tenido en promedio 42,2 $\pm 22,5$ años, mientras que las mujeres $41,0 \pm 19,2$ años. La distribución según grupos de edad y sexo se observa en la Figura 3.

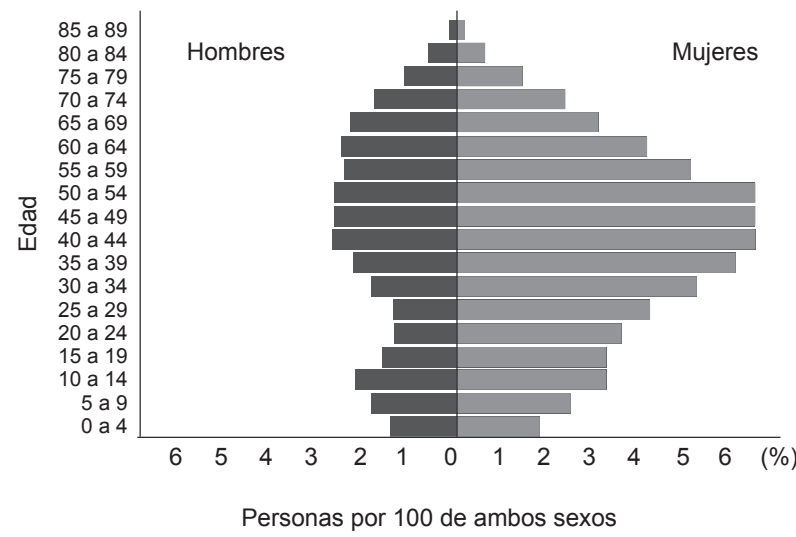

Figura 3. Distribución de los participantes en las campañas "El Día de Lunar", 1995-2011, según edad y sexo.

Fuente de los datos: Círculo Dermatológico del Perú (CIDERM). 
De las casi 120000 personas atendidas, en el 2,8\% se identificó alguna lesión cutánea sospechosa de cáncer de piel, y entre estas lesiones la distribución porcentual fue carcinoma basoceluar $64,9 \%$, melanoma cutáneo $26,7 \%$ y carcinoma espinocelular $8,4 \%$. Las atenciones durante las diferentes campañas han sido realizadas exclusivamente por médicos dermatólogos, quienes ante la identificación de pacientes con una lesión sospechosa de cáncer de piel, lo derivaban a establecimientos del Ministerio de Salud, la Seguridad Social o entidades privadas, de acuerdo a la elección de la persona examinada. Todos los asistentes, además, recibieron consejería sobre las medidas de prevención que deben practicar frente a la exposición solar, y una cartilla de instrucción para el autoexamen de piel e identificación de lesiones sospechosas.

Respecto a los antecedentes de cáncer de piel, el 1,4\% de los participantes refirieron haber sido diagnosticados previamente de alguna neoplasia cutánea, mientras que el 6,0\% tuvo algún familiar con tal diagnóstico. Como es de esperarse, el antecedente personal de cáncer de piel fue más frecuente a mayor edad; así, entre los participantes menores de 20 años este antecedente estuvo presente en solo el $0,5 \%$, entre los de 20 y 39 años en un $0,5 \%$, entre los de 40 y 59 años en 1,4\%, mientras que las personas de 60 años y más presentaron tal antecedente en un 3,5\%. Estos datos no deben ser tomados como una prevalencia de la enfermedad, puesto que al ser el "Día del Lunar" una campaña de salud, es razonable suponer que personas con algún tipo de antecedente o lesión sospechosa acudan con mayor frecuencia. Sin embargo, el dato sí es consistente con la información epidemiológica que señala que a mayor edad, mayor la frecuencia de las neoplasias cutáneas, lo que está también relacionado al efecto acumulativo de la exposición a la radiación ultravioleta.

Otro aspecto que destacar es la evolución del uso de fotoprotectores en la población atendida, el año 2005 (10) el $22,5 \%$ de los participantes refirió su uso $(16,3 \%$ entre los hombres y $25,9 \%$ en las mujeres), mientras que el $2011{ }^{(11)}$ alcanzó el 31,4 \% (22,7\% en hombres y 35,9\% en mujeres). Si bien el porcentaje es aún bajo, se evidencia un incremento sostenido, el cual podría ser un indicador que la población está tomando mayor conciencia de la protección ante la exposición solar.

\section{REFLEXIONES FINALES}

Este 2013 se realizará la XIX campaña del "Día del Lunar" y es importante lo que se ha avanzado a la fecha. No solo por el número de personas atendidas, la cantidad de lesiones sospechosas identificadas y sus características, sino también por la información oral e impresa brindada, con lo cual se cumple con los objetivos de educar y fomentar una cultura de prevención protegiendo a la población, especialmente a los niños, quienes constituyen el segmento más vulnerable. Las campañas también sirven para recopilar información de lo que sucede en la población y el grado de conocimiento que tienen sobre el tema. Los datos referentes a las lesiones sospechosas de cáncer de piel son consistentes con lo descrito a nivel nacional y mundial, de manera que los datos estadísticos recopilados constituyen un buen indicador de la situación de estas neoplasias.

El mensaje que se transmite contiene dos conceptos fundamentales. El primero se refiere a la fotoprotección: debe evitarse sobre exponerse al Sol entre las $10.00 \mathrm{~h}$ y las $16.00 \mathrm{~h}$ y emplear elementos protectores como sombreros, ropa y lentes adecuados, sombrillas y toldos tupidos o gruesos, y de ser posible aplicarse un fotoprotector treinta minutos antes de salir y reaplicarse cada una o dos horas, según la actividad deportiva o laboral a realizar. El segundo corresponde a la detección temprana de lesiones sospechosas: se enseña el ABCDE de los lunares para que la población realice un autoexamen y acuda al médico tempranamente en caso de identificar una lesión sospechosa.

Existen indicadores indirectos que sugieren que andamos por buen camino. Por ejemplo, cada vez es más frecuente, que el paciente acuda al dermatólogo para solicitar una evaluación de sus lunares, además de otras consultas, y solicite información sobre cuál es la mejor manera de protegerse, o lo motive la aparición de una lesión sospechosa. Lo es también el hecho que la prensa toque este tema, sobre todo en verano, y se aprecie a la gente en sus actividades diarias utilizando elementos de fotoprotección o estos sean administrados en sus centros laborales. Desde hace unos años, el Servicio Nacional de Meteorología e Hidrología del Perú (SENAMHI) publica diariamente el índice de radiación ultravioleta y debido a ello algunas municipalidades están instalando en sus distritos "solmáforos" para mantener informada a la población. Algunos colegios están protegiendo a los niños empleando sombreros y evitando realizar al aire libre actividades prolongadas a las horas punta o aplicándoles fotoprotectores. En Arequipa, por ejemplo, se ha aprobado como política regional la implementación de acciones y medidas de protección para reducir los impactos negativos de la radiación ultravioleta mediante Ordenanza Regional 175-Arequipa (12); mientras que en Puno, mediante Ordenanza Regional 005-2012-GRP-CRP, es obligatorio el uso de sombreros y gorros por alumnos y profesores de escuelas públicas y privadas ${ }^{(13)}$. 
Finalmente, en años recientes, otras instituciones médicas afines al tema están realizando actividades paralelas de prevención y detección de cáncer de piel. Si bien esto ha sido posible en gran medida gracias al impulso y el esfuerzo sostenido que los dermatólogos peruanos mantenemos hace 19 años, todavía falta mucho por hacer. Es fundamental entonces seguir difundiendo el mensaje y crear conciencia en las autoridades para que participen activamente en la realización de estos objetivos, como es el caso del Ministerio de Salud y la
Seguridad Social con su apoyo decidido a la campaña del "Día del Lunar". Hay que seguir educando a la población, solicitando a todas las personas y colegas que se sumen coordinadamente a este esfuerzo para seguir avanzando y mejorar lo logrado en beneficio de nuestro país.

Fuentes de financiamiento: autofinanciado.

Conflictos de interés: los autores declaran no tener conflictos de interés.

\section{REFERENCIAS BIBLIOGRÁFICAS}

1. Addison W. Healing gods of ancient civilizations (1925). Whitefish: Kessinger Publishing Co; 2003.

2. Biosalud. Las virtudes terapéuticas de la luz solar. Helioterapia [Internet]. Zaragoza: Instituto Europeo de Biomedicina; 2009 [citado el 14 de enero del 2013]. Disponible en: http://www.biosalud.org/index2. php?sec $=2 \&$ rid $=140$

3. Lucas R, McMichael T, Smith W, Armstrong B. Solar ultraviolet radiation: global burden of disease from solar ultraviolet radiation. Ginebra: Organización Mundial de la Salud; 2006.

4. Sambandan DR, Ratner D. Sunscreens: An overview and update. J Am Acad Dermatol. 2011;64(4):748-58.

5. Rabe JH, Mamelak AJ, McElgunn PJ, Morison WL, Sauder DN. Photoaging: mechanisms and repair. J Am Acad Dermatol. 2006,55(1):1-19.

6. International Agency for Research on Cancer. GLOBOCAN 2008. Estimated cancer
Incidence, Mortality, Prevalence and Disability-adjusted life years (DALYs) Worldwide in 2008 [Internet]. Lyon: OMS; c2010 [citado el 14 de enero de 2013]. Disponible en: http://globocan.iarc.fr/

7. Ramos W. Informe de situación del cáncer a nivel nacional. Informe No 60-2012-GT ENT-DVE-DSVSP/DGE. Lima: Dirección General de Epidemiología, Minsa; 2012.

8. American Academy of Dermatology. Melanoma trends [Internet]. Washington, DC: AAD; c2013 [citado el 16 de enero de 2013]. Disponible en: http://www.aad. org/media-resources/stats-and-facts/conditions/melanoma-trends

9. Actividades del CILAD-PERU. Folia Dermatol Peru. 1995;6(2).

10. Galarza V, Gutiérrez C. Informe final "El día del Lunar". Campaña de educación, prevención y detección temprana del Cáncer de piel y melanoma - 2005. Folia Dermatol Peru. 2005;16(3):107-12.
11. Gutiérrez C. Informe estadístico "El día del Lunar". Campaña de educación, prevención y detección temprana del Cáncer de piel y melanoma - 2011. Folia Dermatol Peru. 2011;22(1):7-9.

12. Ordenanza Regional No 175-Arequipa. Política Regional "Implementación de acciones y medidas de protección para reducir los impactos negativos de la radiación ultravioleta”. Consejo Regional de Arequipa. 07 de agosto de 2012.

13. Ordenanza Regional № 005-2012-GRPCRP. Consejo Regional de Puno. 22 marzo del 2012.

Correspondencia: Carlos E. Sordo Veramatus Dirección: Av. Javier Prado Este 1038, Lima 27. Perú.

Teléfono: (511) 994615936

Correo electrónico: csordo@speedy.com.pe 